\title{
Demonstration of an optical switch based on SOA-MZI operation at $10 \mathrm{Gbit} / \mathrm{s}$
}

\author{
Yalin Guan \\ College of Information Eng ineering \\ Communication University of China \\ Beijing, China \\ 123495518@qq.com
}

\author{
Ruidong Wang \\ College of Information Eng ineering \\ Communication University of China \\ Beijing, China \\ wangruidong3306@gmail.com
}

\begin{abstract}
—it was demonstrated that an optical switch which was operated at $10 \mathrm{Gbit} / \mathrm{s}$ was realized using SOA's in a Mach-Zehnder(SOA-MZI) configuration, which based on cross-phase modulation (XPM) in semiconductor optical amplifier(SOA) and interference struct. An analysis was presented to describe the phase changing in SOA, which caused by optical power injected into SOA. The interference struct supplied a method to transform phase shift into amplitude. At this process, the switch window would been created. Through the simulation the switch was approved.
\end{abstract}

Keywords- Optical switch; MZI; SOA

\section{INTRODUCTION}

All-optical switches are expected to be key devices for various optical communication networks. It is applicabble to ultra-fast optical signal processing such as demultie xing, regenerating, and wavelenght-converting. The device can be realized in various ways for instance using passive or active nonlinear optical components. However, the most widely investigated active nonlinear optical component is the semiconductor optical amplifier (SOA). SOA have attracted much attention for all-optical switching applications, due to their high nonlinearity, simple structure and easy to integrate.

All-optical switching based on nonlinear optical effects in an SOA relies on the application of cross-phase modulation (XPM), cross-gain modulation (XGM) or four-wavemixing (FWM). For high-speed optical switching, the application of XGM is not appropriate, in particular for applications like demultiplexing or add-drop-multiplexing, due to the limited switching contrast. Therefore, In this paper we utilized SOA's in a Mach-Zehnder(SOA-MZI) configuration to realize the optical switch, which based on XPM in SOA and interference struct operation at $10 \mathrm{Gbit} / \mathrm{s}$. the SOA-MZI is a promising candidate as all optical switch because of the inherent stability of its interferometer arrangement and the intrinsically very fast response time based on the XPM in SOA, which enables ultrafast signal processing. Firstly, a phase shift formu la was presented, according to it, phase shift could be achieved by adjusted the injected power. And then phase changing was transferred into amplitude by interference struct, resulted in a switch window created. The width of switching window was controlled by delay time. At last, the switch was approved by the simulation.

\section{OPERATION PRINCIPLE}

An optical switch based on cross-phase modulation in SOA is realized by incorporating the SOA in an interferometer configuration, in which the phase modulation is transferred into an amplitude modulation. the schematic of the SOA-MZI struct was shown in Fig .1. The input siganal beam was split into two beams by a $y$-juntion at the input. The two beams propagated through semiconductor amplifiers located in the two arms of the interferometer. These beams then merged and interfered at an output y-junction. The phase of the interference was altered by injecting another signal in one of the other ports. This phenomenon could be used of functional application. The ratio of the output signal $\left(\mathrm{P}_{0}\right)$ to the input $\operatorname{signal}\left(\mathrm{P}_{\mathrm{i}}\right)$ was given by

$\frac{\mathrm{P}_{\mathrm{o}}}{\mathrm{P}_{\mathrm{i}}}=\frac{1}{8}\left\{\mathrm{G}_{1}+\mathrm{G}_{2}-2 \sqrt{\mathrm{G}_{1} \mathrm{G}_{2}} \cos \left(\emptyset_{1}-\emptyset_{2}\right)\right\}$

Where $G_{1}, G_{2}$ were the gains of the amplifiers and $\emptyset_{1}, \emptyset_{2}$ were phase changes induced bynonlinear effects in the two amplifiers. The phase difference, $\Delta \emptyset=\emptyset_{1}-$ 
$\emptyset_{2}$, at the output was given by

$$
\Delta \emptyset=\frac{\alpha}{2 \mathrm{P}_{\mathrm{s}}}\left\{-\frac{\mathrm{h} v}{\mathrm{e}}\left(\mathrm{I}_{1}-\mathrm{I}_{2}\right)+\frac{\mathrm{P}_{\mathrm{c}}}{2}\left(\mathrm{G}_{1}-1\right)\right\}
$$

Where $\alpha$ was the linewidth enhancement factor. $\mathrm{P}_{\mathrm{c}}$ was the injected power in one of the amplifiers, $P_{s}$ was the saturation power in the amplifiers. For $\mathrm{I}_{1} \cong \mathrm{I}_{2}$ and $\mathrm{G}_{1} \cong \mathrm{G}_{2}=\mathrm{G}$ the above reduced to

$$
\frac{\mathrm{P}_{0}}{\mathrm{P}_{\mathrm{i}}}=\frac{\mathrm{G}}{2} \sin ^{2}\left(\frac{\Delta \emptyset}{2}\right)
$$

Where $\Delta \emptyset=\frac{\alpha}{4} \frac{\mathrm{P}_{\mathrm{c}}}{\mathrm{P}_{\mathrm{s}}}\left(\mathrm{G}_{1}-1\right)$.

through the formula, $\pi$ phase shift could be achieved by adjusted the injected power. There maybe some thermally induced refractive index changes, but these were considerably smaller than the carrier induced changes considered above and could be ignored.

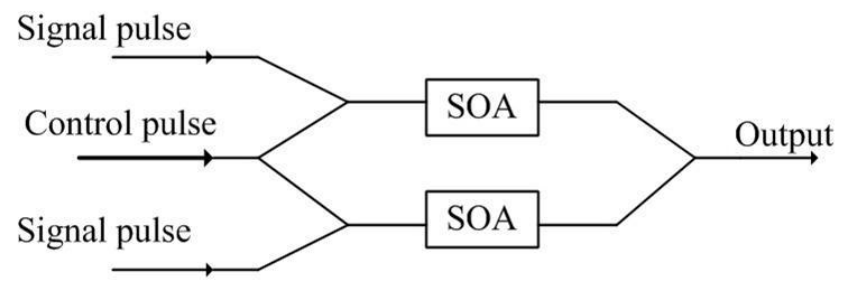

Fig. 1 Schematic of a MZI interferometer integrated with SOA

The schematic of the switch was shown in Fig. 2 . The pump and probe signal were pluse trains with the wavelenghs $\lambda_{\text {punm }}$ and $\lambda_{\text {probe }}$, respectively. Both pluse trains were coupled into the switch with a relative time delay $\Delta t$, which resulted in different widths of switching window. The pulse of pump signal created the switching window, it width was determined in general by the pulse width of pump signal. After switched, the transmitted probe signal were detected by an optical spectrum(OSA)[1-2].

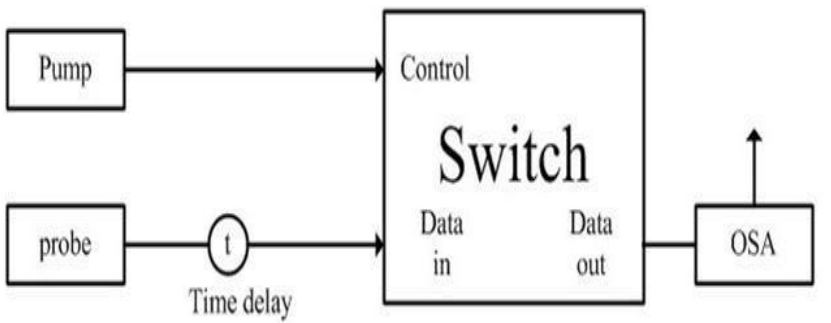

Fig. 2 Operat ing principle of the switch

\section{EXPERIMENT DEMONSTRATION}

The switch simulation was depicted in Fig.3. The SOA-MZI struct had two X-couplers at the input and one at the output, which ere both interconnected by two interferometer arms. Each interferometer arm contained an SOA, the injected current of each SOA were $500 \mathrm{~mA}$. The data signal with a wavelengh $1542 \mathrm{~nm}$ was split and injected into both arms of the interferometer. The two arms were balanced such that the data signal emerged equal at the two outputs of X-coupler without a control pulse. The control pulse with a wavelengh $1555 \mathrm{~nm}$ was split into two pulses, which were coupled into the SOA with a relative time delay $t$, it was achieved by a optical delay. Both control pulse and data pulse were operated at $10 \mathrm{Gbit} / \mathrm{s}$. Each control pulse induced a fast change and a subsequent slow recovery of the refractive index in the SOA. The data pulses experienced this change of the refractive inde $\mathrm{x}$ as a corresponding change of the phases. Under the condition that the phase changing $\Delta \emptyset=\pi$, the data signals in the two arms interfered constructively and tansformed phase changing into amplitude at the output[3-4].

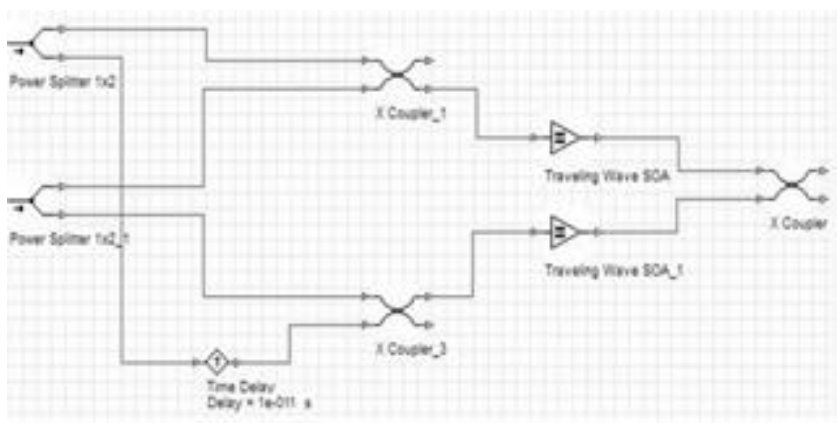

Fig. 3 Simulated the switch based on SOA-MZI

The result of operation was shown in Fig.4. it was clear to see that the control pulse created a switching window successfully and data signal was transmitted, which was depicted in Fig.4(c). The pulse depicted in Fig.4(d) called complementary switching window, it was used for add-drop multip lexing.

\section{CONCLUSION}

SOA-MZI struct was utilized to realiz a $10 \mathrm{Gbit} / \mathrm{s}$ optical switch in this paper. Through the theoretical analysis, the phase shift could be obtained by adjusting the injected pulse power. Using the interferometer, phase 
shift was transferred into amplitude. When the result was proven, the resulting switching window was nearly symmetric and had a width determined by the relative time delay $\mathrm{t}$, because the differential phase shift was nearly zero outside $t$ by proper adjustment of the various device parameters. In the ideal case of a differential phase shift $\Pi$, the data pulse was completely switched. So, more rearch will be done to implement this, and take into account other factors effect on switching window.

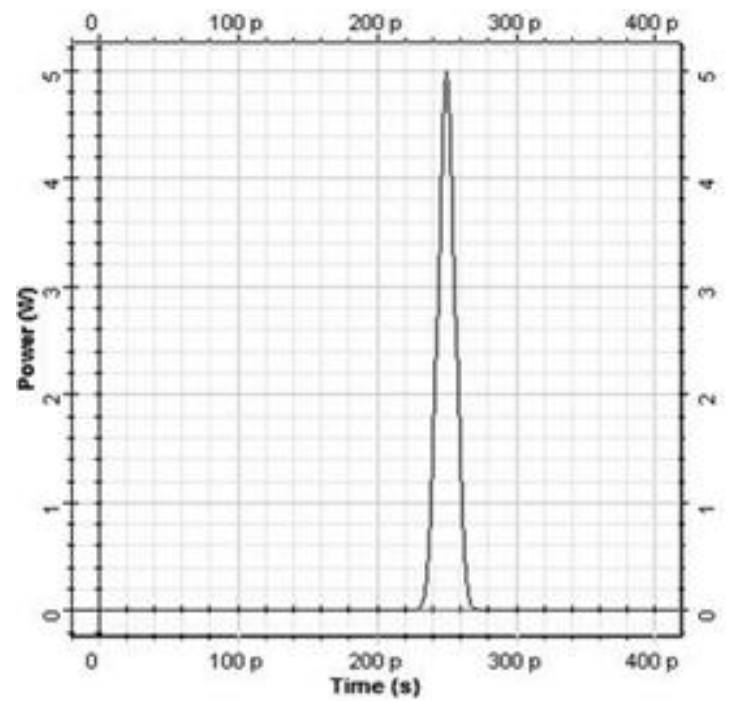

(a)Control signal pulse

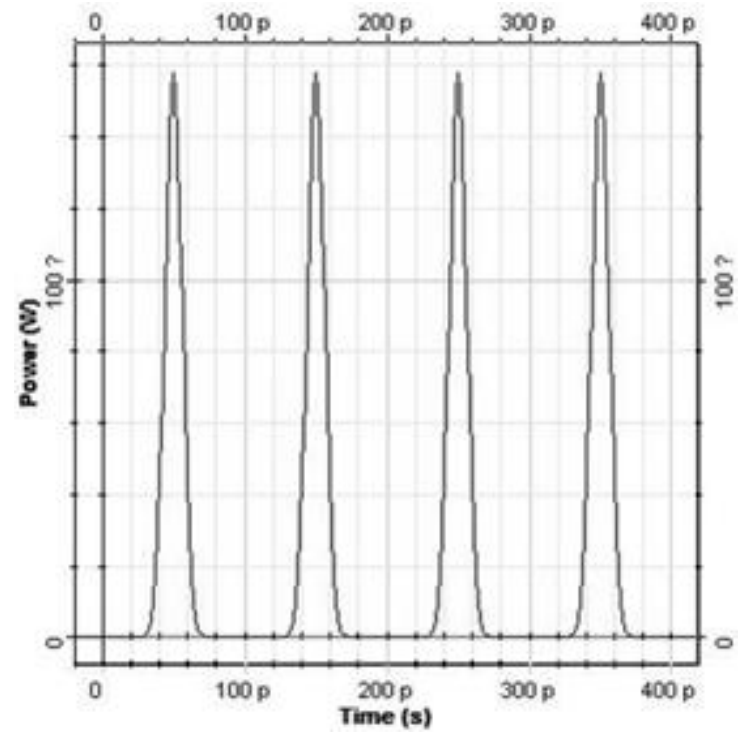

(b) Data signal pulse

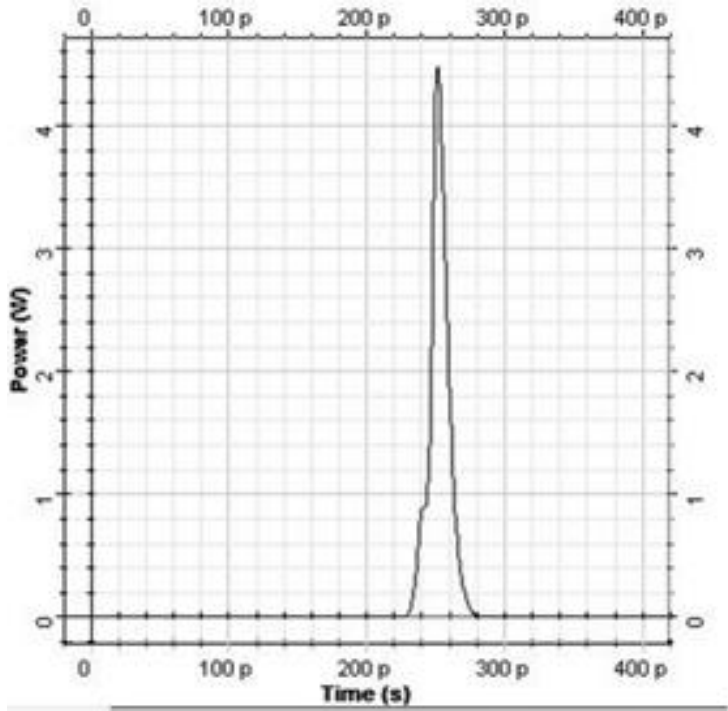

(c) switching window

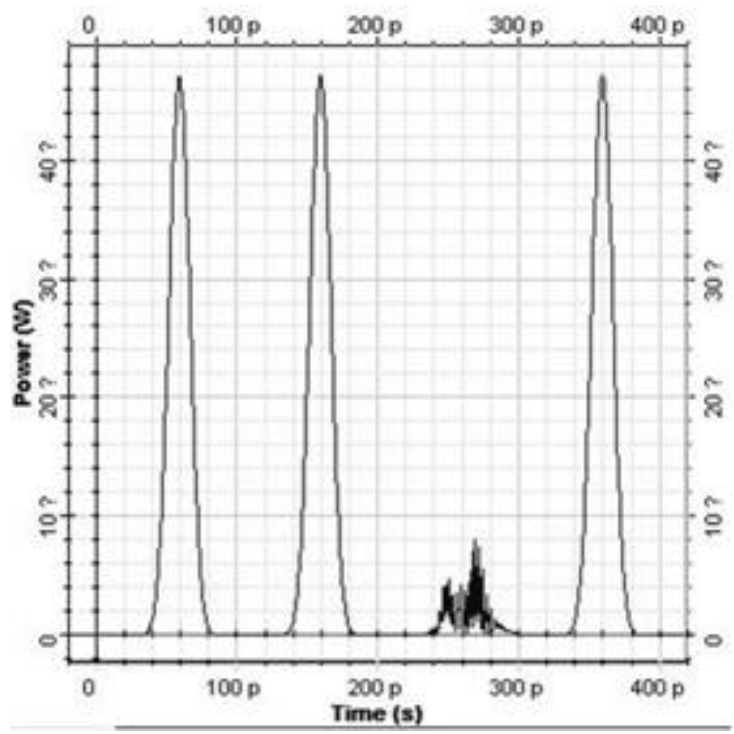

(d) complement ary switching window

Fig. 4 Result for the switch based on SOA-MZI

\section{REFERENCES}

[1] Hans-Georg Weber, Masatka Nakazawa. Ultrahigh-speed Optical Transmission Technology.

[2 ] Niloy K Dutta, Qiang Wang. Semiconduct or Optical Amplifier.

[3] P.-A. Besse, H. Melchior. All-Optical Switches based on Mach-Zehnder Configuration with Improved Extinction Ratios. IEEE PHOTONICS TECHNOLOGY LETTERS, VOL9, NO.1, JANUARY 1997.

[4] G.L. Papadimitriou. Papazoglou and A.S. Pomportsis, Optical Switching: Switch Fabrics, Techniques, and Architectures, IEEE J. Light wave Technol, 2003, 21(2): 384-405. 